\title{
Proportions of Proinflammatory Monocytes Are Important Predictors of Mortality Risk in Hemodialysis Patients
}

\author{
Yachung Jeng, ${ }^{1,2}$ Paik Seong Lim, ${ }^{3,4,5}$ Ming Ying Wu, ${ }^{3}$ Tien-Yu Tseng, ${ }^{3}$ Chang Hsu Chen, ${ }^{3}$ \\ Hung Ping Chen, ${ }^{3}$ and Tsai-Kun $\mathrm{Wu}^{3}$ \\ ${ }^{1}$ Division of Biostatistics and Epidemiology, Department of Medical Research, Tungs' Taichung MetroHarbor Hospital, \\ Taichung, Taiwan \\ ${ }^{2}$ Department of Internal Medicine, National Taiwan University Hospital, Taipei, Taiwan \\ ${ }^{3}$ Division of Renal Medicine, Tungs' Taichung MetroHarbor Hospital, Taichung, Taiwan \\ ${ }^{4}$ Department of Internal Medicine, Taipei Medical University, Taipei, Taiwan \\ ${ }^{5}$ Department of Rehabilitation, Jenteh Junior College of Medicine, Nursing and Management, Miaoli, Taiwan
}

Correspondence should be addressed to Paik Seong Lim; jamespslim@gmail.com

Received 7 May 2017; Revised 6 August 2017; Accepted 13 September 2017; Published 22 October 2017

Academic Editor: Arbi Pecani

Copyright (C) 2017 Yachung Jeng et al. This is an open access article distributed under the Creative Commons Attribution License, which permits unrestricted use, distribution, and reproduction in any medium, provided the original work is properly cited.

\begin{abstract}
Despite the continuous progression in dialysis medicine, mortality and the burden of cardiovascular disease (CVD) among hemodialysis patients are still substantial. Substantial evidence suggests that proinflammatory (CD16+) monocytes contribute to the development of atherosclerosis. A cohort of 136 stable hemodialysis patients (follow-up: 6.25 year) was assessed to investigate the association between the proportion of CD16+ monocytes for all-cause and CVD mortalities. The CD16+ monocytes were associated with both mortalities after adjusting for a preexisting CVD history. Compared to the reference group (CD16+ monocytes within [15.6-18.6], the first and second quartile), patients with CD16+ monocytes above the highest quartile level (>21.5) had an adjusted hazard ratio (HR) of 30.85 (95\% confidence interval [CI]: 7.12-133.8) for CVD mortality and 5.28 (2.07-13.49) for all-cause mortality, and those with CD16+ monocytes below the lowest quartile $\leq 15.6)$, had significantly elevated death risks after 3.5-year follow-up (HR [95\% CI]: 10.9 [2.42-48.96] and 4.38 [1.45-13.24] for CV and all-cause mortalities, respectively). The hemodialysis patients with CD16+ monocyte level in a low but mostly covering normal range also portended a poor prognosis. The findings shed some light for nephrologists on future prospects of early recognizing immune dysfunction and improving early intervention outcomes.
\end{abstract}

\section{Introduction}

It has been established beyond any doubt that cardiovascular (CV) events are an important cause of death, accounting for up to $40-50 \%$, in end-stage renal disease (ESRD) patient population. In the early 70 s, Foley et al. have reported that mortality from cardiovascular disease (CVD) is 10-20 times higher in ESRD patients compared with the general population [1]. Interestingly, some authors found that mortality from non-CV disease in dialysis patients was also increased to the same extent as mortality from CVD [2,3]. Over these years, the potential link between CV and non-CV mortality was explored. Ishani et al. [4] showed that septicemia or bacteremia in dialysis patients was associated with subsequent CV-related events such as myocardial infarction, heart failure, and stroke. On the other hand, the risk of myocardial infarction and that of stroke were substantially higher after a diagnosis of systemic respiratory tract infection [5]. These studies suggested that both CV and infectious causes of death are linked to inflammation, and possibly, these two events may aggravate each other.

Mounting evidence shows that disturbed endothelial function may be an early marker of atherosclerotic process [6]. Clinical and experimental data support a link between endothelial dysfunction and inflammation [7-10]. Chronic systemic inflammation, a common feature in dialysis 
patients, has been identified as an epidemiologically important risk factor for $\mathrm{CV}$ morbidity and mortality in dialysis patients [11-13]. Of 30 prevalent patients, $50 \%$ had elevated serum levels of inflammatory markers such as C-reactive protein, IL-6, and procalcitonin $[12,14,15]$. In addition, a shift towards proinflammatory monocyte subsets [16] and monocyte dysfunction [17] is also noted in these patients. Available evidence showed that even low-grade systemic inflammation has been found to be associated with devastating prognosis of dialysis patients [18-21].

Monocytes can be subdivided into three phenotypically and functionally distinct subpopulations based on the expression of the lipopolysaccharide (LPS) receptor (CD14) and the CD16 (Fcgamma receptor III) $[22,23]$. In healthy individuals, approximately $80-90 \%$ of monocytes are highly CD14 positive and CD16 negative (CD14++CD16-): classical monocytes. The remaining $10-20 \%$ of monocytes are CD16 positive, which are further subdivided into CD14+ +CD16+ and CD14+CD16++ cells, intermediate and nonclassical monocytes, respectively [23]. Compared with CD16 negative conventional monocytes, CD16 positive monocytes, also called proinflammatory monocytes, express higher levels of major histocompatibility complex (MHC) class II antigens, adhesion molecules, chemokine receptors, and proinflammatory cytokines such as TNF- $\alpha$, but lower levels of the anti-inflammatory cytokine, that is, IL-10 [24, 25]. CD16 positive monocytes are elevated in various pathologic conditions, including inflammatory and infectious diseases [26], cancer [27], and in coronary heart disease as ESRD [16, 28, 29]. However, to date, the mechanism by which CD16 positive monocytes increase remains unclear.

Here, we examined the interrelationships between the proportion of proinflammatory monocytes (CD16+ monocytes) and all-cause mortality as well as CV mortality in a cohort of stable ESRD patients on hemodialysis. This study might shed more light on the potential mechanisms that link microinflammation with future CV events.

\section{Methods}

2.1. Patients and Study Sample. Adult outpatients on hemodialysis at the Tungs' Taichung MetroHarbor Hospital (TTMHH) in June 2009 were enrolled. A total of 136 patients were eligible. All the enrolled patients signed informed consents. This study was conducted in full compliance with the provisions of the Personal Information Protection Act and the Human Subjects Research Act of Taiwan and was approved by the institutional review board (number: 102011).

All the patients were dialyzed three times a week with a high-flux polysulfone membrane (FX80 and FX100; Fresenius Medical Care, Bad Homburg, Germany) and bicarbonate dialysate solutions. The median blood flow rate was $280 \mathrm{ml} / \mathrm{min}$ (range $250-300 \mathrm{ml} / \mathrm{min}$ ). All dialysate flows were $800 \mathrm{ml} / \mathrm{min}$, and treatment time was 240 minutes for each patient. All patients were dialyzed through a native arteriovenous (AV) fistula. Blood samples were obtained just before the midweek dialysis session. The dialysate revealed concentrations of bacterial and endotoxin contamination below the detection limit (100 colony-forming units/ml and $<0.25$ endotoxin units). Systolic and diastolic blood pressures (SBP and DBP) were measured in a supine position and after at least a 10-minute rest using a full automatic noninvasive sphygmomanometer.

Each patient's medical chart prior to study enrollment was thoroughly reviewed, and data pertaining to underlying kidney disease, history of CVD, and common comorbid conditions were extracted. The causes of renal failure were diabetic nephropathy $(n=68)$, chronic glomerulonephritis $(n=30)$, polycystic kidney disease $(n=3)$, hypertensive nephrosclerosis $(n=15)$, or unknown $(n=20)$. Patients who had started on hemodialysis for less than 3 months had history of chronic liver diseases, neoplasm, or inflammatory diseases, and those on long-term corticosteroids were excluded. A preexisting history of CVD was defined as a history of coronary artery disease (CAD, including a history of myocardial infarction, coronary artery angioplasty/stenting/ bypass surgery, and carotid endarterectomy/stenting), cerebrovascular disease (CeVD, e.g., stroke), nontraumatic lower extremity amputation, and lower limb artery bypass surgery/ angioplasty/stenting. Diabetes mellitus (DM) cases were ascertained if a patient had a history of DM diagnosis, a spontaneous plasma glucose level of $>200 \mathrm{mg} / \mathrm{dl}$, and/or received hypoglycemic treatment. The survival data were then retrieved in September 2016.

2.2. Laboratory Methods. All blood samples were collected during the midweek dialysis from the AV fistula, immediately after the insertion of the dialysis cannula but before the administration of heparin. Blood was sampled in 4 c.c. Venoject II tubes and centrifuged (10 min, $3000 \mathrm{rpm})$ and stored at $-70^{\circ} \mathrm{C}$ pending analyses, if not analyzed immediately. Serum albumin, urea, creatinine $(\mathrm{Cr})$, total cholesterol, and triglyceride (TG) were determined according to standard methods. The serum levels of high-sensitivity C-reactive protein (hsCRP) were measured using a Behring Nephelometer II (Dade Behring, Tokyo, Japan).

\subsection{Determination of CD14 and CD16 Mononuclear} Phenotype. Peripheral blood was collected by venipuncture using ethylenediaminetetraacetic acid (EDTA) as an anticoagulant. For cytometric analysis, monoclonal antibodies against CD14 (fluorescein isothiocyanate (FITC) conjugated; clone RMO52; Beckman Coulter, Miami, FL, USA), CD16 (phycoerythrin (PE) conjugated; clone 3G8; Beckman Coulter, Miami, FL, USA), CD45 (phycoerythrin cyanin-5 (PC5); clone J33; Beckman Coulter, Miami, FL, USA), and CD56 (clone IM2073; Beckman Coulter, Miami, FL, USA) were used. Briefly, 1001 of the whole blood was stained with saturating amounts of the abovementioned monoclonal antibodies and corresponding isotype controls. After incubation for $15 \mathrm{~min}$ at room temperature in the dark according to the manufacturer's recommendations, OptiLyse C (Beckman Coulter, Miami, FL) was added to lyse RBC and the samples were fixed. Fixed cells were analyzed by flow cytometry within 6 hours.

Determination of leukocyte and monocyte subset distribution was performed using a FC500-Cytometer (Beckman 
Coulter), and CXP analysis software (version 2.2) was used (Schroers et al., 2005). Monocytes were identified as CD45 positive and CD56 negative cells exhibiting a specific forward and sideward scatter profile. Monocytes were then gated in an SSC/CD dot plot, identifying monocytes as CD86 cells with monocyte scatter properties. Subsets of CD14 monocytes with and without CD16 were defined according to the surface expression pattern of the lipopolysaccharide receptor CD14 and the CD16 (Fcgamma receptor III). One million cells were analyzed from each sample, and the percentage of CD16 positive mononuclear cells $(\mathrm{CD} 14+/ \mathrm{CD} 16+$ and $\mathrm{CD14++/CD16+)}$ and the number of cells out of the total monocytes were compared using fluorescent microbeads (Flow-Count, Beckman Coulter). The CD86 antibody (clone HA5.2B7; Beckman Coulter, Miami, FL, USA) was used in this study.

2.4. Statistical Analysis. The sample characteristics were summarized using frequencies and percentage for categorical variables and using median (i.e., the second quartile, $q_{2}$ ), interquartile interval (IQI, an interval bounded by the first and the third quartiles, $q_{1}$ and $q_{3}$ ), mean, and standard deviation (SD) for continuous variables. Spearman's correlation analysis was applied to evaluate bivariate associations between $\mathrm{CD} 16+$ monocytes and other observed variables. The Cox regression was applied to evaluate the association of mortality with $\mathrm{CD} 16+$ monocytes and with other variables. Two types of mortalities were investigated in this study: $\mathrm{CV}$ and all-cause mortalities. The starting point of the survival time was designed at 2009/06/01. Cases who survived till 2016/09/01, transferred to other centers or transplanted during study observation period, were censored at the date. The raw CD16+ monocytes were categorized according to its three quartiles $\left(q_{1}, q_{2}\right.$, and $\left.q_{3}\right)$ into a variable of four levels (from the lowest to the highest level: Q1, Q2, Q3, and Q4). The Cox regression analysis results were displayed in hazard ratio (HR), its associated $95 \%$ confidence interval (CI), and $p$ value. The crossover pattern of hazards among the four-level CD16+ monocytes was modeled using time-dependent effect in the Cox regression model. Throughout this study, tests for statistical associations were evaluated at a significance level of 0.05 . The analyses were all performed in SAS version 9.1.

\section{Results}

The descriptive statistics of the whole study sample were summarized in Table 1 . Of the 136 patients, 39 died in CVD, 18 died in other causes, 8 censored because of transplantation or transferred to another center, and 71 survived till the follow-up ends. The mean (minimum-maximum) follow-up time was $5.57(0.10-7.25)$ years for the overall sample and was $7.03(2.66-7.25)$ years for the 79 nondeath cases.

The Kaplan-Meier curves of CV death and of all-cause death by CD16+ monocyte level were displayed in Figure 1, where follow-up details on the observed case numbers were listed below the figures. The curves overall appeared that patients with $\mathrm{CD} 16+$ monocytes in the fourth quarter had the worst survival rate (the black dotted line in Figure 1)
TABLE 1: The summary of the sample characteristics $(n=136)$.

\begin{tabular}{|c|c|c|}
\hline Variables & $\begin{array}{l}\text { Missing } \\
\text { number }\end{array}$ & $n(\%)$ \\
\hline Sex, female versus male & 0 & $\begin{array}{c}62 \text { (45.59) versus } \\
74(54.41)\end{array}$ \\
\hline DM, no versus yes & 0 & $68(50)$ versus $68(50)$ \\
\hline Hypertension, no versus yes & 0 & $\begin{array}{c}38(27.94) \text { versus } \\
98(72.06)\end{array}$ \\
\hline Preexisting CVD, no versus yes & 3 & $\begin{array}{c}62(46.62) \text { versus } \\
71(53.38)\end{array}$ \\
\hline HD vintage, year & 0 & \\
\hline $0-3$ & & $20(14.71)$ \\
\hline$>3, \leq 5$ & & $37(27.21)$ \\
\hline$>5, \leq 8$ & & $37(27.21)$ \\
\hline \multirow[t]{2}{*}{$>8$} & & $42(30.88)$ \\
\hline & & Median (IQI) \\
\hline CD16+ monocytes, \% & 1 & $18.6(15.6,21.5)$ \\
\hline HD vintage, year & 0 & $6.04(3.92,9.04)$ \\
\hline Age, year & 0 & $59(51.5,69)$ \\
\hline BMI, kg/m² & 0 & $23.62(21.08,25.32)$ \\
\hline $\mathrm{WBC}, 10^{3} / \mathrm{mm}^{3}$ & 1 & $6.6(5.4,7.5)$ \\
\hline Monocyte, $10^{3} / \mu 1$ & 1 & $5.9(4.8,7)$ \\
\hline AbsoMono, cells/ $\mu \mathrm{l}$ & 1 & $367.2(291.2,482.4)$ \\
\hline HsCRP, mg/l & 2 & $2.8(1.5,3.8)$ \\
\hline $\mathrm{Hb}, \mathrm{g} / \mathrm{dl}$ & 1 & $11.2(10.1,12.2)$ \\
\hline PLT, $10^{4} / \mathrm{cm}^{3}$ & 1 & $160(54,218)$ \\
\hline FBS, mg/dl & 1 & $96(82,139)$ \\
\hline $\mathrm{HbA1c}$ & 2 & $6(5,7.1)$ \\
\hline Albumin, g/dl & 1 & $4.2(4,4.4)$ \\
\hline Ferritin, $\mu \mathrm{g} / \mathrm{dl}$ & 2 & $679(467,838)$ \\
\hline $\mathrm{TG}, \mathrm{mg} / \mathrm{dl}$ & 1 & $115(81,187)$ \\
\hline $\mathrm{HDL}, \mathrm{mg} / \mathrm{dl}$ & 2 & $43.5(34,56)$ \\
\hline Cholesterol, mg/dl & 3 & $163(140,189)$ \\
\hline rTG & 3 & $0.77(0.48,1.13)$ \\
\hline rHDL & 3 & $0.28(0.21,0.36)$ \\
\hline cHDL & 3 & $116(94,142)$ \\
\hline BUN, mg/dl & 2 & $66.5(58.1,75.7)$ \\
\hline $\mathrm{Cr}, \mathrm{mg} / \mathrm{dl}$ & 2 & $10.5(9.2,11.9)$ \\
\hline $\mathrm{UA}, \mathrm{mg} / \mathrm{dl}$ & 2 & $7.6(6.7,8.5)$ \\
\hline $\mathrm{Ca}, \mathrm{mg} / \mathrm{dl}$ & 2 & $9.4(9,9.8)$ \\
\hline $\mathrm{P}, \mathrm{mg} / \mathrm{dl}$ & 3 & $4.6(3.8,5.6)$ \\
\hline $\mathrm{SBP}, \mathrm{mmHg}$ & 0 & $138.5(121.5,154.5)$ \\
\hline $\mathrm{DBP}, \mathrm{mmHg}$ & 0 & $77(69,84.5)$ \\
\hline
\end{tabular}

Note: the numbers in the second column indicated the missing numbers of each variable. DM: diabetes mellitus; CVD: cardiovascular diseases including $\mathrm{CAD}$ and CeVD; CAD: coronary artery diseases; CeVD: cerebrovascular disease; BMI: body mass index; WBC: white blood cell count; AbsoMono: absolute monocyte; HsCRP: high-sensitivity C-reactive protein; $\mathrm{Hb}$ : hemoglobin/haemoglobin; PLT: platelet count; FBS: fasting blood sugar; HbAlc: glycated hemoglobin; TG: triglyceride; HDL: high-density lipoprotein cholesterol; cholesterol: total cholesterol; rTG: ratio of TG to cholesterol; rHDL: ratio of HDL to total cholesterol; cHDL: the value resulted from subtracting the level of HDL from the level of total cholesterol; BUN: blood urea nitrogen; Cr: creatinine; UA: uric acid; Ca: serum calcium; P: serum phosphorus; SBP: systolic blood pressure; DBP: diastolic blood pressure; IQI: interquartile interval which is bounded by the first and the third quartiles $\left(q_{1}\right.$ and $\left.q_{3}\right)$ of the variable listed in the first column. 


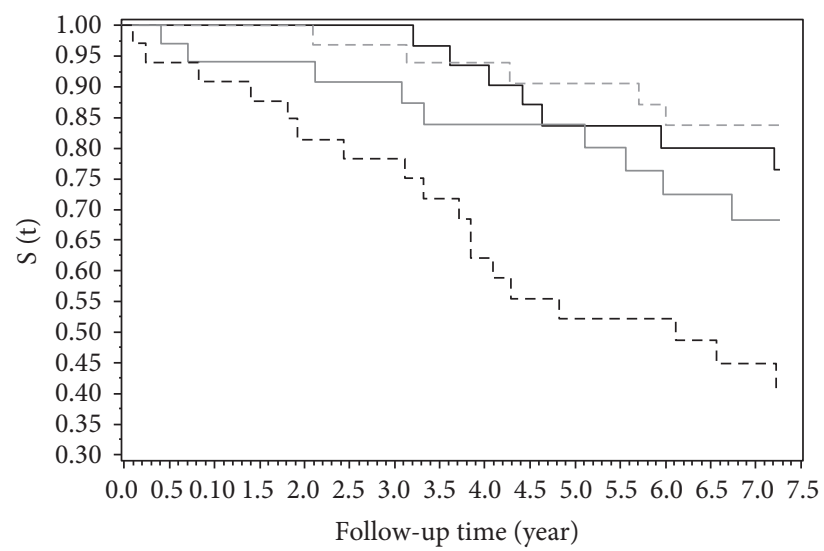

CD16+ monocytes, \%

- 1st quarter: 6.5-15.6 — 3rd quarter: 18.7-21.5

- - 2nd quarter: 15.7-18.6 - - - 4th quarter: 21.6-29.2

\begin{tabular}{lcccccccccccccccc}
\hline Follow-up years & 0 & 0.5 & 1 & 1.5 & 2 & 2.5 & 3 & 3.5 & 4 & 4.5 & 5 & 5.5 & 6 & 6.5 & 7 & 7.5 \\
\hline Number death by CVD history & & & & & & & & & & & & & & & & \\
Present & 0 & 3 & 2 & 1 & 2 & 2 & 0 & 6 & 4 & 5 & 2 & 1 & 3 & 2 & 1 & 1 \\
$\quad$ Nonpresent & 0 & 0 & 0 & 0 & 0 & 1 & 0 & 0 & 0 & 0 & 0 & 0 & 1 & 0 & 1 & 1 \\
Number of survivor & 136 & 133 & 131 & 130 & 128 & 125 & 125 & 119 & 115 & 110 & 108 & 107 & 103 & 101 & 99 & 97 \\
\hline
\end{tabular}

(a) Estimation for CV death $(\log -\operatorname{rank} p$ value $=0.0004)$

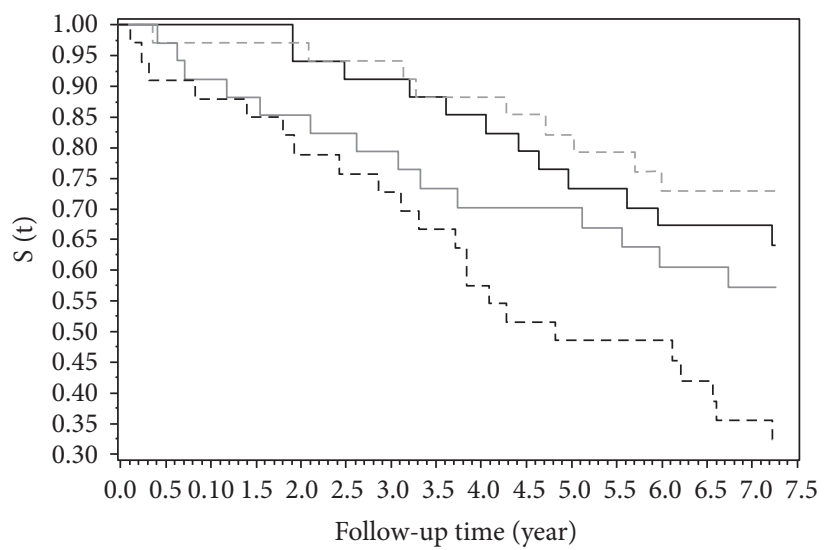

CD16+ monocytes, \%

- 1st quarter: $6.5-15.6$

2nd quarter: $15.7-18.6$

- 3rd quarter: $18.7-21.5$

- - - 4th quarter: 21.6-29.2

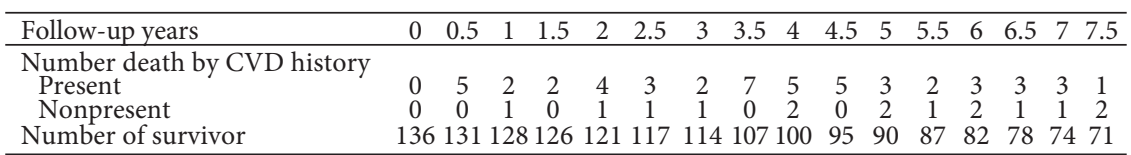

(b) Estimation for all-cause death $(\log$-rank $p$ value $=0.0032)$

FIgURE 1: The Kaplan-Meier curves by CD16+ monocyte level. The Kaplan-Meier curves for CD16+ monocyte level within the lowest to the highest quarters were indicated by black solid line, gray dashed line, gray solid line, and black dashed line. The CD16+ monocyte ranges of the four quarters were the same as those listed in the second row of Table 1. The numbers of death by baseline CVD status and survivor during follow-up were listed below the figures.

and those in the second quarter had better survival rate (the gray dashed line in Figure 1) compared to others. Patients without preexisting CVD history accounted a minor proportion in the overall death numbers: $10.26 \%(4 / 39)$ for $\mathrm{CV}$ death and $23.08 \%(15 / 65)$ for all-cause death. Such numbers were reduced to $5.41 \%(2 / 37)$ and $12.73 \%(7 / 55)$ in subsequent Cox regression analyses because of missing covariates. Since the survival curves for CD16+ monocytes in the first and second quarters were a crossover at 3.5 years, the timedependent effect between the two levels of CD16+ monocytes was incorporated in the later Cox regression for CVD and all-cause mortalities.

The bivariate analysis for CD16+ monocytes and other variables was displayed in Table 2. Most variables were not significantly associated with CD16+ monocyte level, except for age, ferritin, and preexisting CVD history at baseline. 
TABLE 2: The results of association analysis for CD16+ monocytes.

\begin{tabular}{|c|c|c|c|c|c|}
\hline \multirow{2}{*}{ Variables } & \multicolumn{4}{|c|}{ CD16+ monocyte level } & \multirow{2}{*}{$p$ value } \\
\hline & $6.5 \sim 15.6$ & $>15.6, \leq 18.6$ & $>18.6, \leq 21.5$ & $>21.5, \leq 29.2$ & \\
\hline \multirow[t]{2}{*}{$n$} & 34 & 34 & 34 & 33 & \\
\hline & $n(\%)$ & $n(\%)$ & $n(\%)$ & $n(\%)$ & \\
\hline \multicolumn{6}{|l|}{ Sex } \\
\hline Female & $15(44.12 \%)$ & $14(41.18 \%)$ & $15(44.12 \%)$ & $18(54.55 \%)$ & 0.374 \\
\hline Male & $19(55.88 \%)$ & $20(58.82 \%)$ & $19(55.88 \%)$ & $15(45.45 \%)$ & \\
\hline \multicolumn{6}{|l|}{$\mathrm{DM}$} \\
\hline No & $20(58.82 \%)$ & $18(52.94 \%)$ & $16(47.06 \%)$ & $13(39.39 \%)$ & 0.083 \\
\hline Yes & $14(41.18 \%)$ & $16(47.06 \%)$ & $18(52.94 \%)$ & $20(60.61 \%)$ & \\
\hline Hypertension & & & & & 0.806 \\
\hline No & $8(23.53 \%)$ & $11(32.35 \%)$ & $11(32.35 \%)$ & $8(24.24 \%)$ & \\
\hline Yes & $26(76.47 \%)$ & $23(67.65 \%)$ & $23(67.65 \%)$ & $25(75.76 \%)$ & \\
\hline \multicolumn{6}{|c|}{ Preexisting CAD or CeVD } \\
\hline No & $22(64.71 \%)$ & $20(58.82 \%)$ & $13(40.63 \%)$ & $7(21.21 \%)$ & $<0.0001^{*}$ \\
\hline Yes & $12(35.29 \%)$ & $14(41.18 \%)$ & $19(59.38 \%)$ & $26(78.79 \%)$ & \\
\hline \multicolumn{6}{|l|}{ HD vintage (year) } \\
\hline $0-3$ & $7(20.59 \%)$ & $4(11.76 \%)$ & $6(17.65 \%)$ & $3(9.09 \%)$ & 0.849 \\
\hline$>3, \leq 5$ & $8(23.53 \%)$ & $10(29.41 \%)$ & $8(23.53 \%)$ & $11(33.33 \%)$ & \\
\hline$>5, \leq 8$ & $6(17.65 \%)$ & $11(32.35 \%)$ & $14(41.18 \%)$ & $6(18.18 \%)$ & \\
\hline \multirow[t]{2}{*}{$>8$} & $13(38.24 \%)$ & $9(26.47 \%)$ & $6(17.65 \%)$ & $13(39.39 \%)$ & \\
\hline & Mean $\pm S D$ & Mean $\pm S D$ & Mean $\pm S D$ & Mean $\pm S D$ & \\
\hline CD16+ monocytes, $\%$ & $12.77 \pm 2.32$ & $16.96 \pm 0.87$ & $20.28 \pm 0.87$ & $24.77 \pm 2.07$ & - \\
\hline HD vintage, year & $7.99 \pm 5.74$ & $6.8 \pm 3.81$ & $6.29 \pm 3.82$ & $6.89 \pm 3.59$ & 0.753 \\
\hline Age, year & $56.03 \pm 12.03$ & $59.91 \pm 11.35$ & $61.59 \pm 13.15$ & $63.45 \pm 10.67$ & $0.004^{*}$ \\
\hline BMI, kg/m² & $23.1 \pm 3.38$ & $23.31 \pm 3.64$ & $23.1 \pm 3.27$ & $25.03 \pm 3.88$ & 0.238 \\
\hline $\mathrm{WBC}, 10^{3} / \mathrm{mm}^{3}$ & $7.08 \pm 2.12$ & $6.28 \pm 1.43$ & $6.53 \pm 1.58$ & $6.32 \pm 1.35$ & 0.245 \\
\hline Monocyte, $10^{3} / \mu 1$ & $5.71 \pm 1.77$ & $5.89 \pm 1.58$ & $6.27 \pm 1.74$ & $6.06 \pm 1.47$ & 0.192 \\
\hline AbsoMono, cells/ $\mu \mathrm{l}$ & $413.94 \pm 214.01$ & $366.77 \pm 117.41$ & $408.65 \pm 149.62$ & $380.39 \pm 120.19$ & 0.961 \\
\hline HsCRP, mg/l & $2.57 \pm 1.42$ & $2.42 \pm 1.46$ & $2.49 \pm 1.41$ & $2.96 \pm 1.34$ & 0.367 \\
\hline $\mathrm{Hb}, \mathrm{g} / \mathrm{dl}$ & $11.47 \pm 1.59$ & $11.39 \pm 1.31$ & $11.15 \pm 1.57$ & $10.97 \pm 1.27$ & 0.135 \\
\hline PLT, $10^{4} / \mathrm{cm}^{3}$ & $143.21 \pm 90.39$ & $124.96 \pm 82.61$ & $163.69 \pm 88.49$ & $153.32 \pm 89.62$ & 0.311 \\
\hline FBS, mg/dl & $110.85 \pm 42.25$ & $111.86 \pm 43.94$ & $126.56 \pm 59.31$ & $114.76 \pm 45.17$ & 0.732 \\
\hline $\mathrm{HbA1c}, \%$ & $6.38 \pm 1.72$ & $5.96 \pm 1.29$ & $6.29 \pm 1.33$ & $6.59 \pm 1.71$ & 0.198 \\
\hline Albumin, $\mathrm{g} / \mathrm{dl}$ & $4.24 \pm 0.22$ & $4.43 \pm 1.45$ & $4.18 \pm 0.32$ & $4.1 \pm 0.29$ & 0.163 \\
\hline Ferritin, $\mu \mathrm{g} / \mathrm{dl}$ & $657.41 \pm 256.8$ & $594.68 \pm 271.22$ & $745.94 \pm 326.2$ & $717.58 \pm 288.81$ & $0.041^{*}$ \\
\hline $\mathrm{TG}, \mathrm{mg} / \mathrm{dl}$ & $144.56 \pm 107.83$ & $132.05 \pm 73.32$ & $154.29 \pm 105.66$ & $141.42 \pm 83.4$ & 0.782 \\
\hline $\mathrm{HDL}, \mathrm{mg} / \mathrm{dl}$ & $47.18 \pm 17.99$ & $48.82 \pm 17.11$ & $48.03 \pm 19.79$ & $43.48 \pm 14.52$ & 0.877 \\
\hline Cholesterol, mg/dl & $168.5 \pm 37.08$ & $165.7 \pm 37.16$ & $161.24 \pm 37.81$ & $164.12 \pm 41.12$ & 0.767 \\
\hline rTG & $0.86 \pm 0.6$ & $0.82 \pm 0.39$ & $0.9 \pm 0.52$ & $0.88 \pm 0.49$ & 0.805 \\
\hline rHDL & $0.29 \pm 0.11$ & $0.31 \pm 0.12$ & $0.3 \pm 0.11$ & $0.27 \pm 0.09$ & 0.757 \\
\hline $\mathrm{cHDL}, \mathrm{mg} / \mathrm{dl}$ & $121.32 \pm 36.73$ & $116.58 \pm 37.82$ & $113.21 \pm 34.76$ & $120.64 \pm 38.17$ & 0.689 \\
\hline BUN, mg/dl & $67.6 \pm 14.69$ & $67.32 \pm 12.52$ & $66.88 \pm 12.84$ & $65.98 \pm 15.42$ & 0.59 \\
\hline $\mathrm{Cr}, \mathrm{mg} / \mathrm{dl}$ & $10.84 \pm 2$ & $10.66 \pm 2.24$ & $10.72 \pm 2.3$ & $10.07 \pm 2.12$ & 0.149 \\
\hline $\mathrm{UA}, \mathrm{mg} / \mathrm{dl}$ & $7.56 \pm 1.49$ & $7.85 \pm 1.35$ & $7.65 \pm 1.25$ & $7.17 \pm 1.93$ & 0.622 \\
\hline $\mathrm{Ca}, \mathrm{mg} / \mathrm{dl}$ & $13.32 \pm 22.21$ & $9.43 \pm 0.39$ & $9.26 \pm 0.65$ & $9.38 \pm 0.79$ & 0.225 \\
\hline $\mathrm{P}, \mathrm{mg} / \mathrm{dl}$ & $4.65 \pm 1.46$ & $4.75 \pm 1.35$ & $4.41 \pm 1.2$ & $4.76 \pm 1.4$ & 0.55 \\
\hline $\mathrm{SBP}, \mathrm{mmHg}$ & $134.91 \pm 22.34$ & $138.85 \pm 19.65$ & $135.79 \pm 15.02$ & $140.88 \pm 25.02$ & 0.446 \\
\hline $\mathrm{DBP}, \mathrm{mmHg}$ & $78 \pm 10.53$ & $76.38 \pm 10.38$ & $76.15 \pm 8.33$ & $75.79 \pm 9.69$ & 0.479 \\
\hline
\end{tabular}

Note: the abbreviations are the same as those denoted in Table $1 .{ }^{*} p$ value of Spearman's association test using the raw data value of CD16+ monocytes and observed variables. 
TABLE 3: The univariate Cox regression analysis results for death risk.

\begin{tabular}{|c|c|c|c|c|c|c|c|c|}
\hline \multirow{2}{*}{$\begin{array}{l}\text { Outcome types } \\
\text { Covariates }\end{array}$} & \multicolumn{4}{|c|}{ CVD } & \multicolumn{4}{|c|}{ All causes } \\
\hline & HR & \multicolumn{2}{|c|}{$95 \% \mathrm{CI}$} & $p$ value & HR & \multicolumn{2}{|c|}{$95 \%$ CI } & $p$ value \\
\hline \multicolumn{9}{|l|}{ CD16+ monocytes, \% } \\
\hline $\mathrm{Q} 1_{(\leq 3.5 \mathrm{y})}$ versus Q2 & 0.45 & 0.05 & 3.96 & 0.469 & 0.92 & 0.27 & 3.09 & 0.888 \\
\hline $\mathrm{Q1} 1_{(>3.5 \mathrm{y})}$ versus Q2 & 5.16 & 0.56 & 47.86 & 0.149 & 2.00 & 0.53 & 7.63 & 0.308 \\
\hline Q3 versus Q2 & 2.18 & 0.73 & 6.52 & 0.161 & 1.86 & 0.81 & 4.31 & 0.145 \\
\hline Q4 versus Q2 & 5.13 & 1.90 & 13.84 & $0.001^{*}$ & 3.44 & 1.58 & 7.48 & $0.002^{*}$ \\
\hline Sex, male versus female & 0.96 & 0.51 & 1.79 & 0.888 & 0.91 & 0.54 & 1.53 & 0.72 \\
\hline Age, years & 1.03 & 1.01 & 1.06 & $0.011^{*}$ & 1.04 & 1.02 & 1.06 & $<0.001^{*}$ \\
\hline HD vintage, years & 0.97 & 0.9 & 1.04 & 0.42 & 0.95 & 0.89 & 1.01 & 0.132 \\
\hline DM, yes versus no & 2.18 & 1.12 & 4.25 & $0.021^{*}$ & 1.87 & 1.09 & 3.2 & $0.023^{*}$ \\
\hline Hypertension, yes versus no & 3.14 & 1.23 & 8.04 & $0.017^{*}$ & 2.16 & 1.09 & 4.27 & $0.027^{*}$ \\
\hline Preexisting CAD, yes versus no & 7.99 & 3.63 & 17.59 & $<0.001^{*}$ & 5.66 & 3.11 & 10.3 & $<0.001^{*}$ \\
\hline Preexisting CeVD, yes versus no & 2.11 & 1.04 & 4.29 & $0.038^{*}$ & 2.05 & 1.14 & 3.67 & $0.016^{*}$ \\
\hline BMI, $\mathrm{kg} / \mathrm{m}^{2}$ & 1.03 & 0.94 & 1.12 & 0.535 & 0.98 & 0.91 & 1.05 & 0.547 \\
\hline $\mathrm{WBC}, 10^{3} / \mu \mathrm{l}$ & 1.16 & 0.97 & 1.4 & 0.112 & 1.13 & 0.97 & 1.32 & 0.113 \\
\hline Monocyte, $10^{3} / \mu \mathrm{l}$ & 1.06 & 0.87 & 1.28 & 0.558 & 1.05 & 0.89 & 1.23 & 0.583 \\
\hline HsCRP, mg/l & 1.49 & 1.15 & 1.91 & $0.002^{*}$ & 1.38 & 1.12 & 1.68 & $0.002^{*}$ \\
\hline FBS, mg/dl & 1.01 & 1 & 1.01 & $0.019^{*}$ & 1.01 & 1 & 1.01 & $0.01^{*}$ \\
\hline rHDL & 4.98 & 0.31 & 80.71 & 0.259 & 4.95 & 0.5 & 48.94 & 0.172 \\
\hline $\mathrm{cHDL}, \mathrm{mg} / \mathrm{dl}$ & 4.98 & 0.31 & 80.71 & 0.259 & 4.95 & 0.5 & 48.94 & 0.172 \\
\hline $\mathrm{HbA1c}, \%$ & 1.26 & 1.05 & 1.52 & $0.015^{*}$ & 1.21 & 1.04 & 1.42 & $0.015^{*}$ \\
\hline $\mathrm{P}, \mathrm{mg} / \mathrm{dl}$ & 1.06 & 0.84 & 1.34 & 0.606 & 1.01 & 0.83 & 1.22 & 0.945 \\
\hline SBP, mmHg & 1.01 & 1 & 1.03 & 0.171 & 1.01 & 1 & 1.02 & 0.181 \\
\hline $\mathrm{DBP}, \mathrm{mmHg}$ & 1.01 & 0.98 & 1.05 & 0.43 & 1.01 & 0.98 & 1.04 & 0.521 \\
\hline AbsoMono, cells/ $\mu \mathrm{l}$ & 1 & 1 & 1 & 0.121 & 1 & 1 & 1 & 0.127 \\
\hline $\mathrm{PLT}, 10^{3} / \mu \mathrm{l}$ & 1 & 1 & 1.01 & $0.042^{*}$ & 1 & 1 & 1.01 & 0.133 \\
\hline Ferritin, $\mu \mathrm{g} / \mathrm{dl}$ & 1 & 1 & 1 & 0.155 & 1 & 1 & 1 & 0.761 \\
\hline $\mathrm{TG}, \mathrm{mg} / \mathrm{dl}$ & 1 & 1 & 1 & 0.675 & 1 & 1 & 1 & 0.334 \\
\hline $\mathrm{HDL}, \mathrm{mg} / \mathrm{dl}$ & 1 & 0.98 & 1.02 & 0.978 & 1 & 0.98 & 1.01 & 0.84 \\
\hline Cholesterol, mg/dl & 0.99 & 0.98 & 1 & 0.121 & 0.99 & 0.98 & 1 & $0.029^{*}$ \\
\hline rTG & 0.86 & 0.44 & 1.68 & 0.654 & 0.82 & 0.46 & 1.43 & 0.48 \\
\hline $\mathrm{Hb}, \mathrm{g} / \mathrm{dl}$ & 0.89 & 0.7 & 1.13 & 0.348 & 0.92 & 0.76 & 1.12 & 0.405 \\
\hline Albumin, g/dl & 0.23 & 0.06 & 0.85 & $0.028^{*}$ & 0.13 & 0.04 & 0.41 & $<0.001^{*}$ \\
\hline BUN, mg/dl & 1 & 0.97 & 1.02 & 0.802 & 0.99 & 0.97 & 1.01 & 0.165 \\
\hline $\mathrm{Cr}, \mathrm{mg} / \mathrm{dl}$ & 0.83 & 0.71 & 0.97 & $0.019^{*}$ & 0.77 & 0.67 & 0.88 & $<0.001^{*}$ \\
\hline $\mathrm{UA}, \mathrm{mg} / \mathrm{dl}$ & 0.89 & 0.72 & 1.1 & 0.274 & 0.86 & 0.73 & 1.01 & 0.073 \\
\hline $\mathrm{Ca}, \mathrm{mg} / \mathrm{dl}$ & 0.97 & 0.8 & 1.17 & 0.734 & 0.96 & 0.76 & 1.21 & 0.732 \\
\hline
\end{tabular}

Note: the abbreviations are the same as those denoted in Table 1. Q1, Q2, Q3, and Q4 denoted the four ascending classes of the categorical variable which was derived from categorizing each covariate by its three quartiles. The values of the three quartiles were listed in Table 1. Rows indicated as Q1 ${ }_{(\leq 3.5 \mathrm{y})}$ versus $\mathrm{Q} 2$ and Q1 $(>3.5$ y) versus Q2 listed the time-varying effect of CD16+ monocytes below the lowest quartile for follow-up time before and after 3.5 years. * indicates $p$ values of less than 0.05 .

Patients having preexisting CVD tended to have a high-level CD16+ monocytes $(p<0.0001)$. The Spearman correlation coefficients ( $p$ value) for CD16+ monocytes with ferritin and with age was, respectively, $0.18(0.0412)$ and 0.25 (0.0039). Both manifested that higher CD16+ monocytes were correlated to higher ferritin and older age.

The univariate Cox regression analysis results in Table 3 showed that patients with medical conditions such as DM, hypertension, and preexisting CAD or CeVD history tended to have higher risk in both $\mathrm{CV}$ death and all-cause death. In specific, patients with DM at baseline had significantly higher CVD death risk $(\mathrm{HR}=2.18,95 \% \mathrm{CI}: 1.12-4.25)$ and those with a preexisting CeVD event had significantly higher allcause death risk ( $\mathrm{HR}=2.05,95 \% \mathrm{CI}$ : 1.14-3.67). Patients of old age and with high level of hsCRP, fasting blood sugar (FBS), and glycated hemoglobin (HbAlc) tended to have 
TABLE 4: The multiple Cox regression analysis results for death risk.

\begin{tabular}{|c|c|c|c|c|c|c|c|c|}
\hline \multirow{2}{*}{$\begin{array}{l}\text { Outcome types } \\
\text { Covariates }\end{array}$} & \multicolumn{4}{|c|}{ CVD death } & \multicolumn{4}{|c|}{ All-cause death } \\
\hline & HR & \multicolumn{2}{|c|}{$95 \% \mathrm{CI}$} & $p$ value & HR & \multicolumn{2}{|c|}{$95 \% \mathrm{CI}$} & $p$ value \\
\hline \multicolumn{9}{|l|}{ CD16+ monocytes, \% } \\
\hline Q4 versus Q3 & 12.81 & 3.72 & 44.09 & $<0.001^{*}$ & 3.26 & 1.49 & 7.14 & $0.003^{*}$ \\
\hline Q4 versus Q2 & 30.85 & 7.12 & 133.8 & $<0.001^{*}$ & 5.28 & 2.07 & 13.49 & $<0.001^{*}$ \\
\hline Q3 versus Q2 & 2.41 & 0.58 & 10.03 & 0.227 & 1.62 & 0.61 & 4.29 & 0.333 \\
\hline \multicolumn{9}{|l|}{ Follow-up time $\leq 3.5$ years } \\
\hline Q1 versus Q2 & 1.63 & 0.15 & 17.33 & 0.685 & 0.9 & 0.26 & 3.16 & 0.875 \\
\hline Q4 versus Q1 & 18.92 & 2.17 & 164.9 & $0.008^{*}$ & 5.84 & 1.71 & 19.98 & $0.005^{*}$ \\
\hline Q3 versus Q1 & 1.48 & 0.16 & 13.47 & 0.73 & 1.79 & 0.52 & 6.17 & 0.358 \\
\hline \multicolumn{9}{|l|}{ Follow-up time $>3.5$ years } \\
\hline Q1 versus Q2 & 10.9 & 2.42 & 48.96 & $0.002^{*}$ & 4.38 & 1.45 & 13.24 & $0.009^{*}$ \\
\hline Q4 versus Q1 & 2.83 & 0.81 & 9.86 & 0.102 & 1.21 & 0.44 & 3.29 & 0.716 \\
\hline Q3 versus Q1 & 0.22 & 0.05 & 0.91 & $0.037^{*}$ & 0.37 & 0.13 & 1.07 & 0.067 \\
\hline \multicolumn{9}{|l|}{ Baseline medical condition } \\
\hline CeVD history, yes versus no & 6.98 & 2.18 & 22.3 & $0.001^{*}$ & 2.74 & 1.41 & 5.32 & $0.003^{*}$ \\
\hline CAD history, yes versus no & 44.57 & 13.1 & 151.7 & $<0.001^{*}$ & 9.4 & 4.53 & 19.48 & $<0.001^{*}$ \\
\hline CAD history versus CeVD history & 6.39 & 1.79 & 22.8 & $0.004^{*}$ & 3.43 & 1.34 & 8.75 & $0.01^{*}$ \\
\hline Age, $>q_{2}$ versus others & 2.88 & 1.14 & 7.28 & $0.025^{*}$ & 2.36 & 1.24 & 4.52 & $0.009^{*}$ \\
\hline Cholesterol, $>q_{2}$ versus others & 2.98 & 1.19 & 7.44 & $0.02 *$ & - & - & - & - \\
\hline Platelet, $>q_{2}$ versus others & 7.23 & 2.66 & 19.67 & $<0.001^{*}$ & 3.99 & 2.04 & 7.79 & $<0.001^{*}$ \\
\hline \multicolumn{9}{|l|}{$\mathrm{Cr}$} \\
\hline$\leq q_{1}$ versus others & 4.26 & 1.82 & 10 & $<0.001^{*}$ & - & - & - & - \\
\hline$\leq q_{2}$ versus others & - & - & - & - & 4.49 & 2.31 & 8.75 & $<0.001^{*}$ \\
\hline $\mathrm{UA}, \leq q_{1}$ or $>q_{3}$ versus others & 7.38 & 2.47 & 22.01 & $<0.001^{*}$ & 2.5 & 1.35 & 4.62 & $0.003^{*}$ \\
\hline $\mathrm{SBP},>q_{2}$ versus others & 5.11 & 1.92 & 13.59 & $0.001^{*}$ & - & - & - & - \\
\hline $\mathrm{DBP}, \leq q_{1}$ or $>q_{3}$ versus others & 7.43 & 2.62 & 21.06 & $<0.001^{*}$ & 4.08 & 1.98 & 8.4 & $<0.001^{*}$ \\
\hline rTG, $>q_{3}$ versus others & 0.18 & 0.07 & 0.47 & $<0.001^{*}$ & 0.21 & 0.09 & 0.47 & $<0.001^{*}$ \\
\hline Ferritin, $>q_{3}$ versus others & - & - & - & - & 0.45 & 0.23 & 0.87 & $0.018^{*}$ \\
\hline
\end{tabular}

Note: the abbreviations are the same as those indicated in Table $1 . q_{1}, q_{2}$, and $q_{3}$ denoted the three quartiles-the first quartile, median, and the third quartile-of each covariate and the values were listed in Table 1. Q1, Q2, Q3, and Q4 here denoted the four ascending categories derived from the categorized CD16+ monocyte levels by its three quartiles. * indicates $p$ values of less than 0.05 .

higher risk in both mortalities (all these variables had HRs $>1$ in Table 3). Those with high level in albumin and Cr tended to have lower risk in both mortalities (all these variables had HRs $<1$ in Table 3). For instance, a CVD death risk $H R$ value of 1.03 (95\% CI: 1.01-1.06) for the variable age in Table 3 indicated that a 1-year increment in age is significantly associated with $3 \%$ increase in CVD death risk. An all-cause death risk HR value of 0.13 (95\% CI: 0.04-0.41) for the variable albumin indicated that $1 \mathrm{~g} / \mathrm{dl}$ increment in albumin is significantly associated with $87 \%$ decrease in all-cause death risk. Patients who had CD16+ monocyte level lying in the second quarter (i.e., CD16+ monocyte level $>q_{1}$ and CD16+ monocyte level $\leq q_{2}$ ) expressed the lowest risks in both CVD and all-cause death (a J-shaped relationship).

Both the Kaplan-Meier curve and the univariate Cox regression analysis demonstrated a J-shaped relationship between CD16+ monocytes and patients' death risks, especially after the time of follow-up exceeds 3.5 years.
Considering the possible effect of the lowest quartile of CD16+ monocytes and the risk of death, any possible reverse causation was adequately addressed in the analyses by maintaining a varying reference category. A multiple Cox regression analysis (Table 4) demonstrated that the J-shaped relationship between CD16+ monocytes and hemodialysis patients' death risks persisted after accounting for baseline conditions, for the hazard crossover effect between the two lowest quarters of CD16+ monocytes, and for a range of covariates. Patients with CD16+ monocytes in the fourth quarter manifested significantly higher death risks as compared to all other quarters; the HRs ranged from 2.83 to 30.85 for CV death and from 1.21 to 5.84 for all-cause death after adjusting other covariates (see Table 4 for detailed results). Further, patients with $\mathrm{CD} 16+$ monocytes below $q_{1}$ had an elevated adjusted HR for both CV death $(\mathrm{HR}=10.9, p=0.002)$ and for all-cause death $(\mathrm{HR}=4.38$, $p=0.009)$ in the fully adjusted model. 
In analysis regarding $\mathrm{CV}$ death risk, preexisting $\mathrm{CeVD}$ and $\mathrm{CAD}$ history had a significant effect after adjusting the effect of $\mathrm{CD} 16+$ monocytes $(\mathrm{HR}=6.98$ and $\mathrm{HR}=44.57$, resp., both $p \leq 0.001)$. Interestingly, a preexisting CAD history appeared to be associated with higher $\mathrm{CV}$ death risk than a preexisting $\mathrm{CeVD}(\mathrm{HR}=6.39, p=0.004)$. Patients of old age (above median), with PLT, SBP, and cholesterol above median, with $\mathrm{Cr}$ below the first quartile, with uric acid (UA) and DBP out of the IQI, and with ratio of TG to total cholesterol (rTG) below the third quartile, were associated with higher $\mathrm{CV}$ death risk. For all-cause death risk, the presence of preexisting CeVD and CAD had a significant effect $(\mathrm{HR}=2.74$ and $\mathrm{HR}=9.4$, resp., $p=0.003$ and $p<0.001)$ after adjusting the effect of CD16+ monocytes. Patients of age above median, with PLT above median, with $\mathrm{Cr}$ below median, with UA and DBP out of the IQI, and with rTG and ferritin below the third quartile, were associated with higher all-cause death risk. The detailed results were listed in Table 4.

\section{Discussion}

Our data accord with some previous findings of increased mortality in dialysis patients with higher percentages of nonclassical CD16 positive monocytes. Recent studies have established that phenotypic variations in the surface of monocytes are associated with the occurrence of CVD in both chronic kidney disease (CKD) and non-CKD patients. While Berg et al. found that classical CD16 negative monocytes can predict future $\mathrm{CV}$ risk in nonuremic population [30], some authors found that intermediate CD14++CD16+ monocytes predict CV events in CKD patients [31, 32]. Differences in study design and studied populations may account for some of the discrepancies regarding the correlation of monocyte subsets and adverse cardiac events in these studies. Nevertheless, flow cytometry is a powerful technique and its use has obviously allowed for risk stratification in a wide variety of diseases.

The innate immune system plays a major role in the initiation and propagation of atherosclerosis, with monocytes/ macrophages being the key component in this process [33]. Apart from being responsible for counteracting exogenous bacterial, viral, and fungal infections [34], they are also involved in endogenous inflammatory processes. They contribute to atherogenesis through promoting leukocyte recruitment to plaques, and their roles are also mediated by activation of downstream signaling pathways, such as nuclear factor kappa-B pathway [35]. Monocyte involvement in the development of atherosclerotic plaques was reported in the 1970s, with monocyte accumulation demonstrated in porcine atherosclerotic lesions [36]. In recent years, we became aware of the role of different monocyte subsets in the pathogenesis of atherosclerosis, particularly specific monocyte subpopulations with their diverse phenotypes and sentinel roles in both the innate and adaptive immune system. Our understanding of how monocyte subsets participate in this process is largely based on mouse models of atherosclerosis $[37,38]$.
In non-CKD populations, many cohort and case-control studies have documented an association of monocytosis with cardiovascular diseases [39-42]. Elevated monocyte counts were also identified as an independent predictor of total and CV mortality in hemodialysis patients [43]. In a cohort of 951 patients, Rogacev et al. [31] found that nonclassical CD14+CD16+ monocytes independently predicted cardiovascular events in subjects referred for elective coronary angiography. Numbers of CD16 positive monocytes but not overall monocyte counts positively correlate with body mass index and insulin resistance as well as diabetes and intimamedia thickness [44]. In patients with symptomatic CAD compared to healthy controls, the percentage of CD16 positive monocytes was found to be increased after adjustment for common risk factors $[45,46]$. Assessment of plaque vulnerability in patients with both stable and unstable angina pectoris found that more vulnerable plaques were associated with an increase in percentage of CD16 positive monocytes.

CKD had been shown to alter the number, subset distribution, and function of circulating monocytes [47, 48]. In previous studies $[16,31,32]$, patients with CKD have an increased percentage of CD16 positive monocytes in the circulation. In our study, we further observed that in patients with preexisting CVD, the presence of higher percentage of CD16 positive monocytes was found to be associated with increased CV and all-cause death.

More interestingly, we found that a subset of dialysis patients with CD16+ monocytes falling within the normal range tends to suffer great risk of $\mathrm{CV}$ death. Advanced $\mathrm{CKD}$ is characterized by the dynamic coexistence of the generalized immune depression that contributes to the high prevalence of infections among these patients and systemic inflammation that may contribute to CVD. Accumulation of proinflammatory cytokines may be due to decreased renal elimination and/or increased generation following induction by various factors such as uremic toxins, oxidative stress, volume overload, and comorbidities $[49,50]$. ESRD is associated with immunosuppression due to the impact of the uremic milieu and a variety of associated metabolic disorders on the other. Impaired monocyte function, including defects in chemotaxis, phagocytosis, and a decrease in the production of cytokines, had been reported [50]. Frequent exposure to these diverse external stimuli might lead to a state of chronic low-grade activation, and a high-percentage monocytic primed cell was found in hemodialysis patients [51].

The prevailing and continuous antigenic stimulation might result in exhaustion in the downstream signaling cascade, and this might subsequently impair the innate and adaptive components of the immune system's response to microbial challenge. The presence of a subgroup of our patients with functional monocyte deactivation may be due to LPS tolerance. This state of "immune paralysis" in these patients may be related to downregulation of toll-like receptor, especially toll-like receptor-2 (TLR-2) and toll-like receptor-4 (TLR-4) expression on monocytes [49, 52]. TLR2 and TLR- 4 are involved in innate immunity, and activation of these receptors leads to systemic inflammation in the host. Several authors found that a decrease of TLR- 4 was found on unstimulated monocytes in CKD patients compared with 
healthy controls [52-54]. The etiological factor of "immune paralysis" may be related to chronic endotoxemia [55], frequent blood membrane interaction, or other toxic metabolites related to uremic milieu. It seems possible that continuous activation of monocytes suppresses the expression of TLR-4, contributing to immune deficiency and increased incidence and severity of infections in ESRD population. Clearly, the J-shaped effect of low CD16+ monocytes on $\mathrm{CV}$ death risk observed in this study needs further research for it to be clarified.

Lastly, the analysis results of this study, namely, the association between CD16+ monocytes and mortalities in hemodialysis patients, were obtained mostly in patients with a preexisting CVD history. In the multiple Cox regression analysis, we found that those without CVD history at baseline had just 2 nonmissing cases suffered from CV death and 7 nonmissing cases from all-cause death. Moreover, the association pattern shown in Table 4 remained unchanged when the analyses were performed on the sample composed of those with CVD history at baseline.

We acknowledge several limitations of this study. One of them is the relatively small number of patients, particularly the relatively small number of patients without preexisting CAD. Thus, caution should be exercised in the interpretation of our results. Besides, our patient population was also limited to those on hemodialysis and may not be generalizable to the broader population.

Taken together, the results of this study indicated that high level in CD16+ monocytes was associated with significantly higher risks in CV and all-cause death in hemodialysis patients with preexisting CAD. Overall, nonclassic monocytes were detrimental, whereas the minor subset of the relatively low CD16-expressing monocytes was associated with an unfavorable clinical outcome. In spite of the limited study sample, we highlight the current facts and future perspectives of how the assessment of microinflammation can assist clinicians in early and efficient recognition of inappropriate performance of the immune system to reduce mortality. Nevertheless, more studies based on large-scale cohort are still desired to elucidate this issue further.

\section{Disclosure}

The funder had no role in the study design, data collection and analysis, decision to publish, or preparation of the manuscript.

\section{Conflicts of Interest}

The authors declare that there is no conflict of interest regarding the publication of this paper.

\section{Acknowledgments}

This study was funded by a grant from Tungs' Taichung MetroHarbor Hospital, Taichung, Taiwan, Grant no. TTMHH-102C0013.

\section{References}

[1] R. N. Foley, P. S. Parfrey, and M. J. Sarnak, "Clinical epidemiology of cardiovascular disease in chronic renal disease," American Journal of Kidney Diseases, vol. 32, no. 5, pp. S112-S119, 1998.

[2] D. J. De Jager, D. C. Grootendorst, K. J. Jager et al., "Cardiovascular and noncardiovascular mortality among patients starting dialysis," The Journal of the American Medical Association, vol. 302, no. 16, pp. 1782-1789, 2009.

[3] M. J. Sarnak and B. L. Jaber, "Pulmonary infectious mortality among patients with end-stage renal disease," Chest, vol. 120, no. 6, pp. 1883-1887, 2001.

[4] A. Ishani, A. J. Collins, C. A. Herzog, and R. N. Foley, "Septicemia, access and cardiovascular disease in dialysis patients: the USRDS wave 2 study," Kidney International, vol. 68, no. 1, pp. 311-318, 2005.

[5] L. Smeeth, S. L. Thomas, A. J. Hall, R. Hubbard, P. Farrington, and P. Vallance, "Risk of myocardial infarction and stroke after acute infection or vaccination," The New England Journal of Medicine, vol. 351, no. 25, pp. 2611-2618, 2004.

[6] T. F. Lüscher and M. Barton, "Biology of the endothelium," Clinical Cardiology, vol. 20, no. 11, Supplement 2, pp. II-3II10, 1997.

[7] G. K. Hansson, "Inflammation, atherosclerosis and coronary artery disease," The New England Journal of Medicine, vol. 352, no. 16, pp. 1685-1695, 2005.

[8] K. Bhagat and P. Vallance, "Inflammatory cytokines impair endothelium-dependent dilatation in human veins in vivo," Circulation, vol. 96, no. 9, pp. 3042-3047, 1997.

[9] S. Fichtlscherer, G. Rosenberger, D. H. Walter, S. Breuer, S. Dimmeler, and A. M. Zeiher, "Elevated C-reactive protein levels and impaired endothelial vasoreactivity in patients with coronary artery disease," Circulation, vol. 102, no. 9, pp. 1000-1006, 2000.

[10] J. Sinisallo, J. Paronen, K. J. Mattila et al., "Relation of inflammation to vascular function in patients with coronary heart disease," Atherosclerosis, vol. 149, no. 2, pp. 403-441, 2000.

[11] V. Menon, T. Greene, X. Wang et al., "C-reactive protein and albumin as predictors of all-cause and cardiovascular mortality in chronic kidney disease," Kidney International, vol. 68, no. 2, pp. 766-772, 2005.

[12] J. Y. Yeun, R. A. Levine, V. Mantadilok, and G. A. Kaysen, "C-reactive protein predicts all-cause and cardiovascular mortality in hemodialysis patients," American Journal of Kidney Diseases, vol. 35, no. 3, pp. 469-476, 2000.

[13] J. Zimmermann, S. Herrlinger, A. Pruy, T. Metzger, and C. Wanner, "Inflammation enhances cardiovascular risk and mortality in hemodialysis patients," Kidney International, vol. 55, no. 2, pp. 648-658, 1999.

[14] V. Panichi, U. Maggiore, D. Taccola et al., "Interleukin-6 is a stronger predictor of total and cardiovascular mortality than C-reactive protein in dialytic patients," Nephrology, Dialysis, Transplantation, vol. 19, no. 5, pp. 1154-1160, 2004.

[15] G. Conti, A. Amore, M. Chiesa et al., "Procalcitonin as a marker of micro-inflammation in hemodialysis," Journal of Nephrology, vol. 18, no. 3, pp. 282-288, 2005.

[16] W. A. Nockher and J. E. Scherberich, "Expanded CD14 $\mathrm{CD}_{16}{ }^{+}$monocyte subpopulation in patients with acute and chronic infections undergoing hemodialysis," Infection and Immunity, vol. 66, no. 6, pp. 2782-2790, 1998. 
[17] S. C. Meuer, M. Hauer, P. Kurz, K. M. ZumBüschenfelde, and H. Köhler, "Selective blockade of the antigenreceptor-mediated pathway of $\mathrm{T}$ cell activation in patients with impaired primary immune responses," The Journal of Clinical Investigation, vol. 80, no. 3, pp. 743-749, 1987.

[18] G. A. Kaysen, "The microinflammatory state in uremia. Causes and potential consequences," Journal of the American Society of Nephrology, vol. 12, no. 7, pp. 1549-1557, 2001.

[19] R. Pecoits-Filho, B. Lindholm, and P. Stenvinkel, "The malnutrition, inflammation, and atherosclerosis (MIA) syndrome the heart of the matter," Nephrology, Dialysis, Transplantation, vol. 17, Supplement 11, pp. 28-31, 2002.

[20] P. Stenvinkel, R. Pecoits-Filho, and B. Lindholm, "Coronary artery disease in end-stage renal disease: no longer a simple plumbing problem," Journal of the American Society of Nephrology, vol. 14, no. 7, pp. 1927-1939, 2003.

[21] Y. Xu, Y. Chen, D. Li et al., "Hypertension, fluid overload and microinflammation are associated with left ventricular hypertrophy in maintenance hemodialysis patients," Renal Failure, vol. 35, no. 9, pp. 1204-1209, 2013.

[22] S. Gordon and P. R. Taylor, "Monocyte and macrophage heterogeneity," Nature Reviews Immunology, vol. 5, no. 12, pp. 953-964, 2005.

[23] L. Ziegler-Heitbrock, P. Ancuta, S. Crowe et al., "Nomenclature of monocytes and dendritic cells in blood," Blood, vol. 116, no. 16, pp. e74-e80, 2010.

[24] N. Kawanaka, M. Yamamura, T. Aita et al., "CD14+, CD16+ blood monocytes and joint inflammation in rheumatoid arthritis," Arthritis \& Rheumatism, vol. 46, no. 10, pp. 25782586, 2002.

[25] H. W. L. Ziegler-Heitbrock, "Heterogeneity of human blood monocytes: the $\mathrm{CD} 14^{+} \mathrm{CD} 16^{+}$subpopulation," Immunology Today, vol. 17, no. 9, pp. 424-428, 1996.

[26] H. Janols, A. Bredberg, I. Thuvesson, S. Janciauskiene, O. Grip, and M. Wullt, "Lymphocyte and monocyte flow cytometry immunophenotyping as a diagnostic tool in uncharacteristic inflammatory disorders," BMC Infectious Diseases, vol. 10, no. 1 , p. $205,2010$.

[27] H. W. Ziegler-Heitbrock, G. Fingerle, M. Strobel et al., "The novel subset of $\mathrm{CD} 14^{+} / \mathrm{CD} 16^{+}$blood monocytes exhibits features of tissue macrophages," European Journal of Immunology, vol. 23, no. 9, pp. 2053-2058, 1993.

[28] M. Nahrendorf, M. J. Pittet, and F. K. Swirski, "Monocytes: protagonists of infarct inflammation and repair after myocardial infarction," Circulation, vol. 121, no. 22, pp. 2437-2445, 2010.

[29] U. Sester, M. Sester, G. Heine, H. Kaul, M. Girndt, and H. Köhler, "Strong depletion of $\mathrm{CD} 14^{+} \mathrm{CD} 16^{+}$monocytes during haemodialysis treatment," Nephrology, Dialysis, Transplantation, vol. 16, no. 7, pp. 1402-1408, 2001.

[30] K. E. Berg, I. Ljungcrantz, L. Andersson et al., "Elevated $\mathrm{CD} 14^{++} \mathrm{CD} 16^{-}$monocytes predict cardiovascular events," Circulation. Cardiovascular Genetics, vol. 5, no. 1, pp. 122131, 2012.

[31] K. S. Rogacev, S. Seiler, A. M. Zawada et al., "CD14 ${ }^{++} \mathrm{CD} 16^{+}$ monocytes and cardiovascular outcome in patients with chronic kidney disease," European Heart Journal, vol. 32, no. 1, pp. 84-92, 2011.

[32] G. H. Heine, C. Ulrich, E. Seibert et al., "CD14 ${ }^{++} \mathrm{CD} 16^{+}$ monocytes but not total monocyte numbers predict cardiovascular events in dialysis patients," Kidney International, vol. 73, no. 5, pp. 622-629, 2008.

[33] M. M. Oude Nijhuis, J. K. van Keulen, G. Pasterkamp, P. H. Quax, and D. P. de Kleijn, "Activation of the innate immune system in atherosclerotic disease," Current Pharmaceutical Design, vol. 13, no. 10, pp. 983-994, 2007.

[34] P. Libby, "Inflammation in atherosclerosis," Nature, vol. 420, no. 6917, pp. 868-874, 2002.

[35] M. P. De Winther, E. Kanters, G. Kraal, and M. H. Hofker, "Nuclear factor $\kappa \mathrm{B}$ signaling in atherogenesis," Arteriosclerosis, Thrombosis, and Vascular Biology, vol. 25, no. 5, pp. 904-914, 2005.

[36] B. A. Kottke and M. T. Subbiah, "Pathogenesis of atherosclerosis. Concepts based on animal models," Mayo Clinic Proceedings, vol. 53, no. 1, pp. 35-48, 1978.

[37] F. K. Swirski, P. Libby, E. Aikawa et al., "Ly-6C hi $^{\text {monocytes }}$ dominate hypercholesterolemia-associated monocytosis and give rise to macrophages in atheromata," The Journal of Clinical Investigation, vol. 117, no. 1, pp. 195-205, 2007.

[38] F. Tacke, D. Alvarez, T. J. Kaplan et al., "Monocyte subsets differentially employ CCR2, CCR5, and CX3CR1 to accumulate within atherosclerotic plaques," The Journal of Clinical Investigation, vol. 117, no. 1, pp. 185-194, 2007.

[39] K. Nasir, E. Guallar, A. Navas-Acien, M. H. Criqui, and J. A. Lima, "Relationship of monocyte count and peripheral arterial disease: results from the National Health and Nutrition Examination Survey 1999-2002," Arteriosclerosis, Thrombosis, and Vascular Biology, vol. 25, no. 9, pp. 1966-1971, 2005.

[40] B. D. Horne, J. L. Anderson, J. M. John et al., "Which white blood cell subtypes predict increased cardiovascular risk?," Journal of the American College of Cardiology, vol. 45, no. 10, pp. 1638-1643, 2005.

[41] R. Dragu, S. Huri, R. Zuckerman et al., "Predictive value of white blood cell subtypes for long-term outcome following myocardial infarction," Atherosclerosis, vol. 196, no. 1, pp. 405-412, 2008.

[42] A. J. Grau, A. W. Boddy, D. A. Dukovic et al., "Leukocyte count as an independent predictor of recurrent ischemic events," Stroke, vol. 35, no. 5, pp. 1147-1152, 2004.

[43] A. Kato, T. Takita, M. Furuhashi, Y. Maruyama, H. Kumagai, and A. Hishida, "Blood monocyte count is a predictor of total and cardiovascular mortality in hemodialysis patients," Nephron Clinical Practice, vol. 110, no. 4, pp. c235-c243, 2008.

[44] C. Poitou, E. Dalmas, M. Renovato et al., "CD14 ${ }^{\mathrm{dim}} \mathrm{CD} 16^{+}$and $\mathrm{CD} 14^{+} \mathrm{CD} 16^{+}$monocytes in obesity and during weight loss: relationships with fat mass and subclinical atherosclerosis," Arteriosclerosis, Thrombosis, and Vascular Biology, vol. 31, no. 10, pp. 2322-2330, 2011.

[45] A. Schlitt, G. H. Heine, S. Blankenberg et al., "CD14+CD16+ monocytes in coronary artery disease and their relationship to serum TNF- $\alpha$ levels," Thrombosis and Haemostasis, vol. 92, no. 2, pp. 419-424, 2004.

[46] M. Wildgruber, H. Lee, A. Chudnovskiy et al., "Monocyte subset dynamics in human atherosclerosis can be profiled with magnetic nano-sensors," PLoS One, vol. 4, no. 5, article e5663, 2009.

[47] W. H. Lim, S. Kireta, E. Leedham, G. R. Russ, and P. T. Coates, "Uremia impairs monocyte and monocyte-derived dendritic cell function in hemodialysis patients," Kidney International, vol. 72, no. 9, pp. 1138-1148, 2007. 
[48] G. H. Heine, A. Ortiz, Z. A. Massy et al., "Monocyte subpopulations and cardiovascular risk in chronic kidney disease," Nature Reviews Nephrology, vol. 8, no. 6, pp. 362-369, 2012.

[49] S. Kato, M. Chmielewski, H. Honda et al., "Aspects of immune dysfunction in end-stage renal disease," Clinical Journal of the American Society of Nephrology, vol. 3, no. 5, pp. 1526-1533, 2008.

[50] S. Gonçalves, R. Pecoits-Filho, S. Perreto et al., "Associations between renal function, volume status and endotoxaemia in chronic kidney disease patients," Nephrology, Dialysis, Transplantation, vol. 21, no. 10, pp. 2788-2794, 2006.

[51] H. W. Kim, Y. S. Woo, H. N. Yang et al., "Primed monocytes: putative culprits of chronic low-grade inflammation and impaired innate immune responses in patients on hemodialysis," Clinical and Experimental Nephrology, vol. 15, no. 2, pp. 258-263, 2011.

[52] M. Koc, A. Toprak, H. Arikan et al., "Toll-like receptor expression in monocytes in patients with chronic kidney disease and haemodialysis: relation with inflammation," Nephrology, Dialysis, Transplantation, vol. 26, no. 3, pp. 955-963, 2011.

[53] M. Ando, A. Shibuya, K. Tsuchiya, T. Akiba, and K. Nitta, "Reduced expression of Toll-like receptor 4 contributes to impaired cytokine response of monocytes in uremic patients," Kidney International, vol. 70, no. 2, pp. 358-362, 2006.

[54] Y. Kuroki, K. Tsuchida, I. Go et al., "A study of innate immunity in patients with end-stage renal disease: special reference to toll-like receptor-2 and -4 expression in peripheral blood monocytes of hemodialysis patients," International Journal of Molecular Medicine, vol. 19, no. 5, pp. 783-790, 2007.

[55] C. Y. Lin, I. F. Tsai, Y. P. Ho et al., "Endotoxemia contributes to the immune paralysis in patients with cirrhosis," Journal of Hepatology, vol. 46, no. 5, pp. 816-826, 2007. 


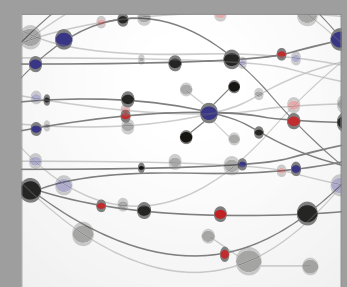

The Scientific World Journal
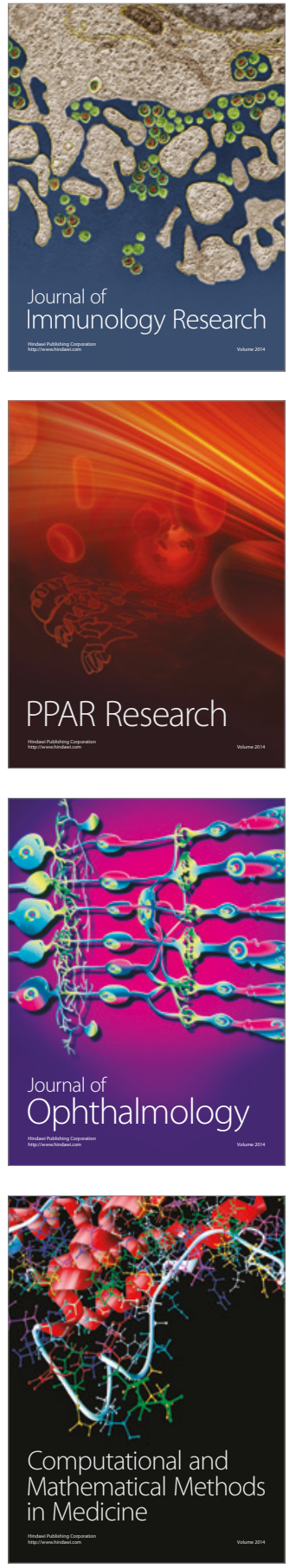

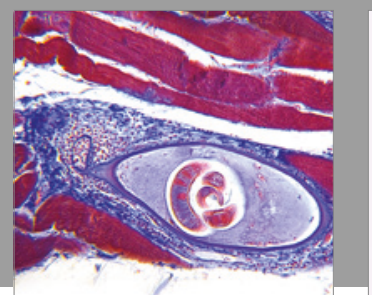

Gastroenterology Research and Practice
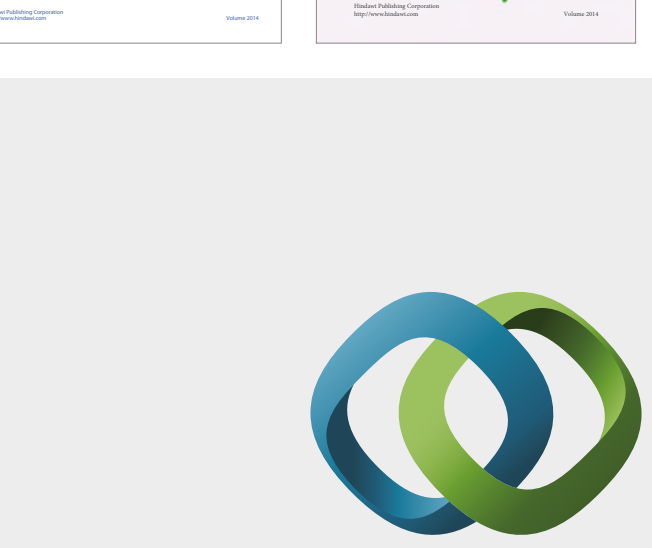

\section{Hindawi}

Submit your manuscripts at

https://www.hindawi.com
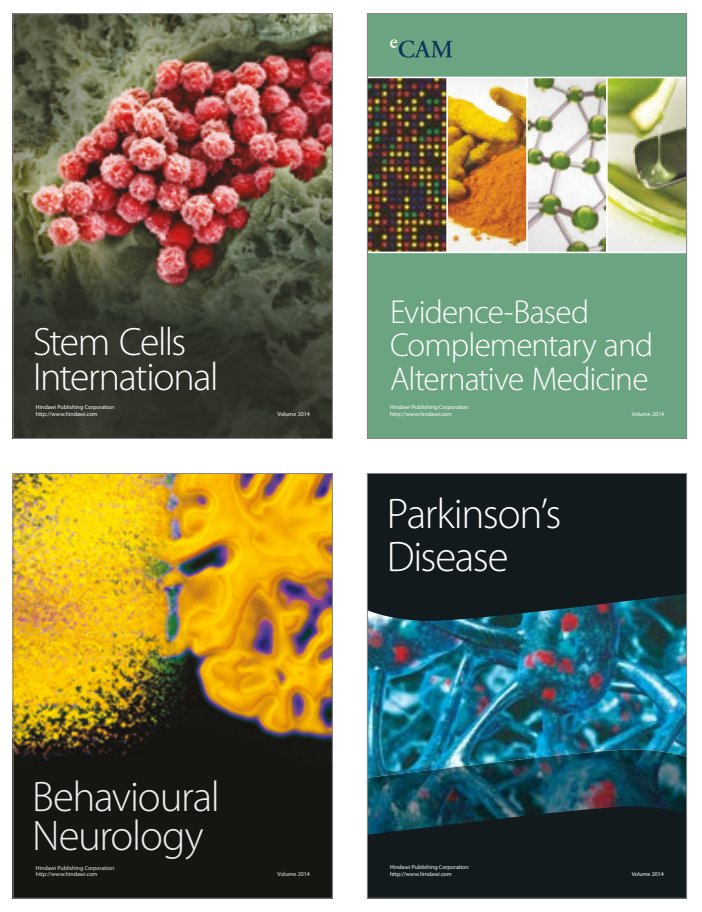
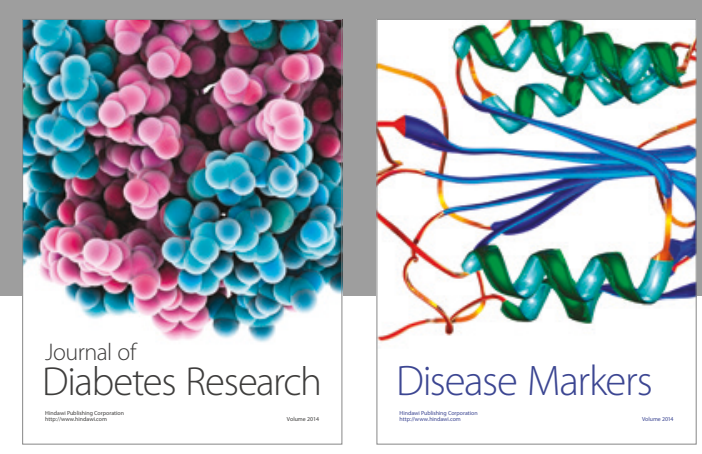

Disease Markers
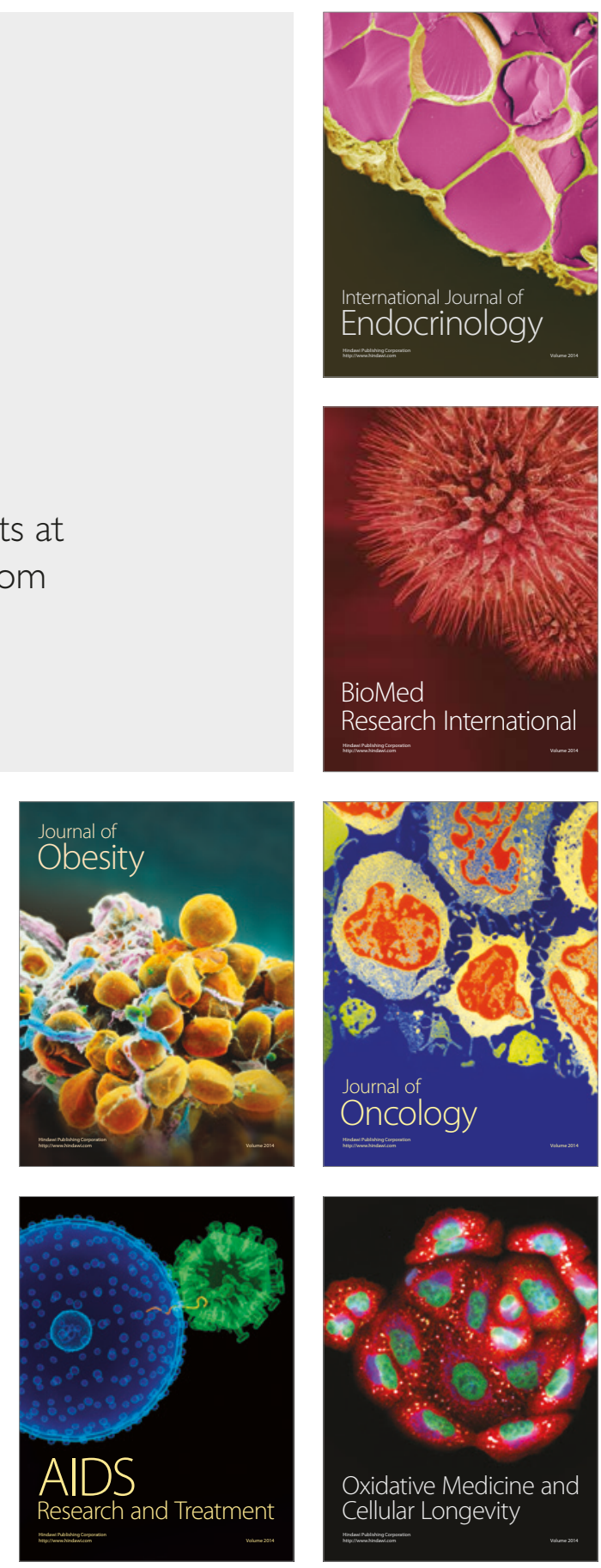